\title{
Emerging themes in medicinal glycoscience
}

\author{
Kathryn M. Koeller and Chi-Huey Wong* \\ Department of Chemistry, The Scripps Research Institute and Skaggs Institute for Chemical Biology, 10550 North Torrey Pines Road, La Jolla, CA 92037. \\ ${ }^{*}$ Corresponding author(wong@Scripps.edu).
}

Received 17 May 2000; accepted 5 July 2000

\begin{abstract}
The recognition of complex carbohydrates and glycoconjugates as mediators of important biological processes has stimulated investigation into their therapeutic potential. New approaches for the simplification of glycoconjugate synthesis are overcoming the limitations of existing methods and providing a diverse array of these biomolecules. As the accessibility of glycoconjugates increases, carbohydratebased constructs are becoming available for analysis as medicinal agents in a wide range of therapies.
\end{abstract}

Keywords: glycoconjugate, synthesis, therapeutic, antibiotic, vaccine

Numerous medicinally relevant physiological events rely on glycoconjugates for their viability ${ }^{1-3}$. The recognition of glycoprocessing enzymes as targets for therapeutic intervention has spurred the development of numerous drug candidates ${ }^{4,5}$, including the well-known "flu" drugs that inhibit influenza virus neuraminidase. Likewise, the discovery of the selectins attracted researchers to the study of carbohydrate-protein interactions in the inflammatory response ${ }^{6}$. The observation that cell surface glycoforms are altered in certain cancers has served as both a diagnostic tool and the foundation of glycoconjugate-based vaccine development ${ }^{7}$. Glycans provide required recognition elements of many antipathogenic agents ${ }^{8}$, and the glycopolymers heparin and hyaluronic acid are employed clinically as anticoagulants ${ }^{9}$ or arthritis therapies, respectively. Furthermore, protein glycosyl phosphatidylinositol (GPI) anchors ${ }^{10}$ and polylactosamine chains ${ }^{11}$ play structural roles on the cell surface, and a nuclear $\beta$ $O$-GlcNAc modification may function in signal transduction pathways ${ }^{12}$. Moreover, the glycoprotein hormone erythropoeitin, used for clinical treatment of anemia, requires sialyl oligosaccharides for optimal activity in vivo ${ }^{13}$.

Examination of the growing repertoire of glycoconjugate function provides assurance that this field will be at the forefront of research for years to come. Therapeutic agents based on carbohydrates or biological pathways in which they are involved have become important pharmaceutical targets. This review highlights areas in which glycoconstructs have been investigated for medicinal applications.

\section{Problems in the development of glycotherapeutics}

Many reliable options are available to protein and nucleic acid scientists for procuring a particular biomolecule. Automated synthesizers allow the manufacture of short peptide and nucleic acid sequences, and expression systems and polymerase chain reaction (PCR) make obtaining large quantities of macromolecular material a trivial consideration. Unfortunately, this is not the case for oligosaccharides and other glycoconjugates.

This discrepancy largely results from the intrinsic structure of carbohydrates themselves. To synthesize peptides or oligonucleotides, a method is available for iterative formation of a single type of bond (i.e., peptide or phosphodiester bond, respectively). However, synthesis of specific glycosidic linkages is a much more difficult task, as carbohydrates are densely functionalized with hydroxyl groups of similar reactivity. Synthetic carbohydrate chemists will attest to the many hurdles encountered in obtaining even relatively simple oligosaccharides, including laborious protecting-group manipulations and long overall synthetic routes. In addition, the degree of molecular diversity that can be generated from glycosidic linkage assembly is enormous: more than 10 million tetrasaccharides can be assembled from the 9 common monosaccharides, compared with 256 tetranucleotides and 16,000 tetrapeptides from the corresponding 4 nucleotides and 20 amino acid building blocks, respectively. Consequently, advancing glycoconjugate synthetic technology to the same level as proteins and nucleic acids remains a daunting challenge.

On a macromolecular scale, various cellular expression systems have proven useful for protein production. Yet, obtaining homogeneous glycoproteins is not a trivial matter. Glycosylation is a posttranslational modification, rather than falling under direct transcriptional control, and glycan structure is therefore subject to several environmental factors. Enzyme competition for the same substrate, enzyme substrate specificity and availability, as well as variant glycosylation patterns with cellular host and culture conditions all play a role in the form of the resultant glycan ${ }^{14}$. Glycoproteins obtained from prokaryotic or eukaryotic expression systems therefore exhibit glycoform microheterogeneity ${ }^{15}$. This makes the contribution of specific glycan structure to underlying protein structure and function nearly impossible to assess.

\section{Newly developed synthetic strategies}

The ongoing investigation of carbohydrate-based therapeutic agents is dependent on the development of reliable synthetic methods to make glycoconjugates more readily available on a large scale. Toward this end, the incorporation of enzymes into synthetic protocols has the potential to drastically reduce the number of protecting-group manipulations and synthetic steps required ${ }^{16-18}$.

Glycosyltransferases, especially combined with regeneration of sugar nucleotides (Fig. 1A), have great synthetic utility in the formation of glycosidic linkages ${ }^{16-18}$. Furthermore, genetic manipulation of the biosynthetic pathways in microorganisms has improved the production of sugar nucleotides ${ }^{19}$. The use of proteases as catalysts in peptide bond formation ${ }^{20}$ and lipases for the mild removal of protecting groups ${ }^{21}$ have been extremely benefi- 
A

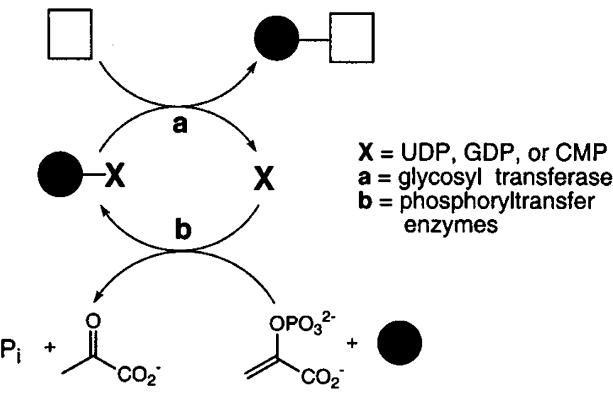

C

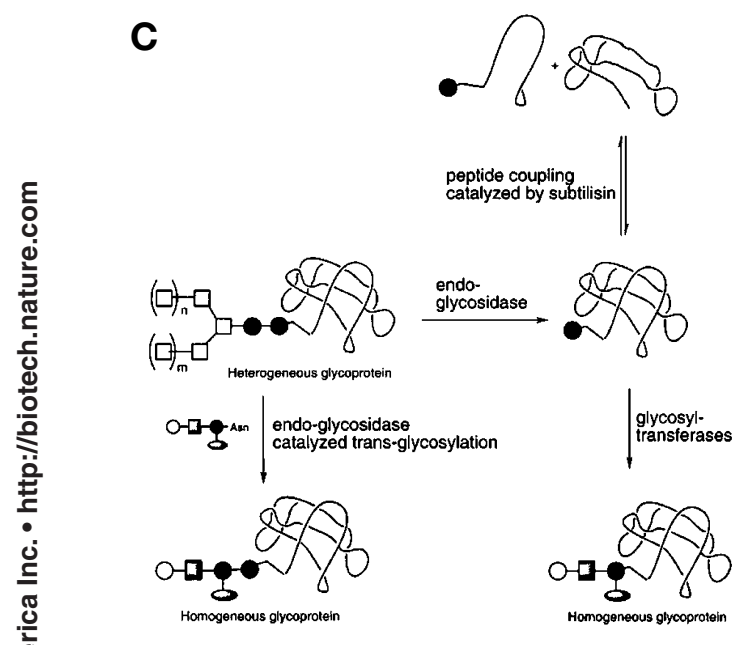

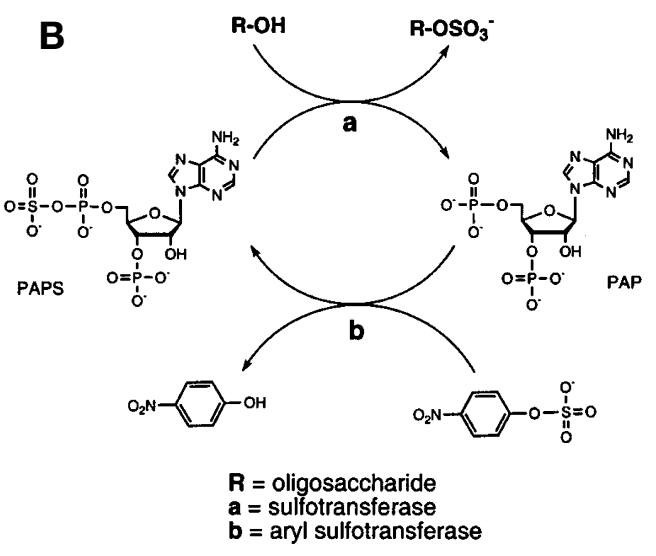

Figure 1. Selected approaches for the synthesis of complex glycoconjugates. (A) The combination of glycosyltransferase-catalyzed reactions and the recycling of sugar nucleotides for the synthesis of complex carbohydrates. (B) The regeneration of PAPS for the sulfation of oligosaccharides and other glycoconjugates. (C) Strategies for the construction of homogeneous glycoproteins using endoglycosidases, proteases, and glycosyltransferases. (D) One-pot programmable method for the synthesis of oligosaccharides.

D
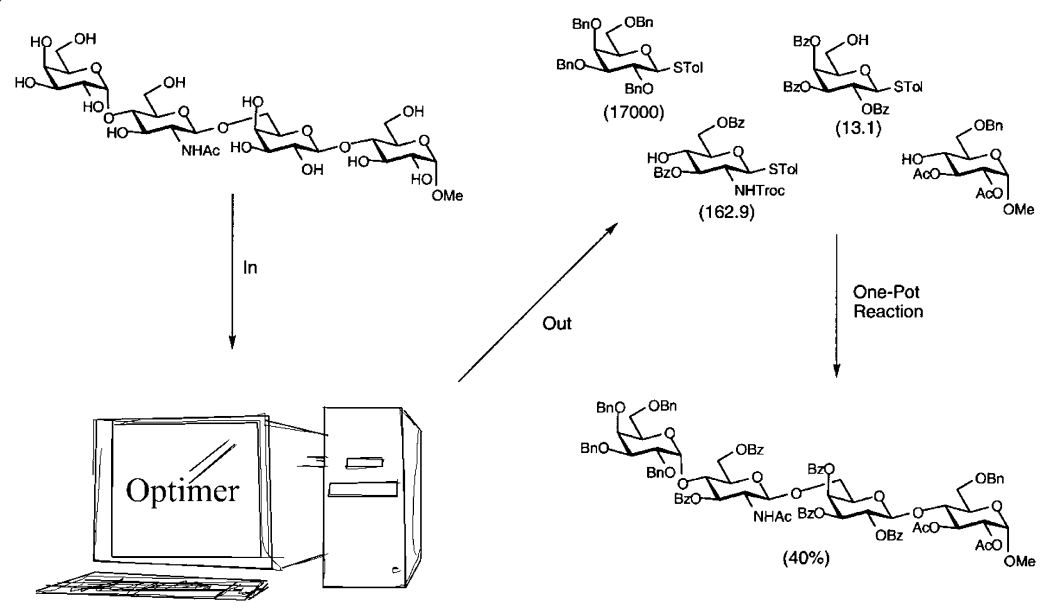

cial in the area of glycopeptide synthesis ${ }^{18}$, as well as for site-specific esterification ${ }^{22}$. Aldolases have provided access to numerous unnatural carbohydrate derivatives ${ }^{16,23}$, and directed evolution is yielding novel catalysts with altered substrate specificity (S. Fong \& C.-H. Wong, unpublished data). New enzymatic protocols for the recycling of $3^{\prime}$-phospho adenosine $5^{\prime}$-phosphosulfate (PAPS) as a sulfotransferase donor substrate have been developed (Fig. 1B) and will further allow elucidation of the role of glycoconjugate sulfation ${ }^{24}$. Endoglycosidases, proteases, and glycosyltransferases can be sequentially employed to produce glycoproteins with homogeneous glycoforms (Fig. 1C) ${ }^{20}$. As methods for the construction of homogeneous glycoproteins are greatly needed, other synthetic avenues, such as native peptide ligation ${ }^{25}$, inteincatalyzed peptide bond formation ${ }^{26}$, and endoglycosidase-catalyzed transglycosylation have also been explored ${ }^{18,27}$ (Fig. 1C). At present, enzymes specific for every desired linkage are not currently available, and strategies combining chemical and enzymatic synthetic methods have been generally advantageous.

On the chemical forefront, many research groups have reported "one-pot" strategies for the synthesis of oligosaccharides ${ }^{18}$. These methods involve the selection of glycosyl donors that will react in a predefined order, thus potentially simplifying the overall synthetic procedure. The culmination of these efforts has resulted in the development of "one-pot programmable" methods (Fig. 1D) ${ }^{28}$, which rely on the ability to control glycosylation reactions by careful selection of hydroxyl protecting groups, the ability to quantify glycosyl donor reactivity, and a universal donor activation strategy. Having already demonstrated its utility in the programmed synthesis of linear and branched oligosaccharides, this method continues to be developed, and represents the nearest precursor to automated carbohydrate synthesis that exists to date.
Although these unique one-pot and enzymatic synthetic methods have proven utility in the generation of glycoconjugates, traditional chemical synthesis continues to be a backbone staple. The following sections outline various approaches to carbohydratebased therapeutics that were possible through chemical and chemoenzymatic synthetic approaches.

\section{Antiviral agents}

The influenza virus is one of the best-known pathogens dependent on carbohydrate-recognition for its infectivity. The viral hemagglutinin protein is responsible for initial recognition of host cell surface sialic acid (NeuAc), which is subsequently cleaved by the viral neuraminidase, allowing infection to proceed ${ }^{29,30}$. Anti-influenza drug design has focused on the inhibition of both hemagglutinin and neuraminidase, though in markedly different strategies (Fig. 2). Hemagglutinin exists as a homotrimer present in multiple copies on the viral cell surface and, in accordance, the most potent hemagglutinin inhibitors are polyvalent constructs $^{31,32}$. Inhibition of neuraminidase, in contrast, has relied on mimicking the transition state of the hydrolytic reaction by rational design. Several of these transition-state analogs are potent neuraminidase inhibitors, and are now on the market ${ }^{33,34}$.

Galactosyl-ceramide (Gal-Cer) on host cells is employed as an alternate receptor for HIV-1, and is a point of attachment for the V3 loop of gp12035. Soluble Gal-Cer analogs have been analyzed as inhibitors of viral infection through disruption of the naturally occurring gp120-Gal-Cer interaction ${ }^{36,37}$. Anti-HIV activity can also be accomplished by interfering with protein-RNA interactions ${ }^{38}$. The neomycin class of aminoglycoside antibiotics binds to the Rev responsive element (RRE) of HIV mRNA, and inhibits the interaction of the RRE with Rev protein ${ }^{39}$. This prevents the switch from viral latency to active viral replication. Synthetic agents that inhibit 
A

\section{B}
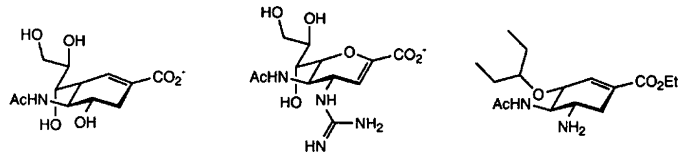

Figure 2. Strategies for the inhibition of influenza virus. (A) Polyvalent hemagglutinin inhibitors. (B) Transition-state analog neuraminidase inhibitors.

RRE-Rev protein interactions with high affinity and specificity are currently being developed as potential antiretroviral agents ${ }^{39,40}$.

\section{Antibacterial and antimicrobial agents}

Many microorganisms rely on the recognition of glycoconjugates for the ability to infect a given host cell ${ }^{41}$. Not surprisingly, antibiotics that target invasive organisms also often contain glycostructure. In this area, a recent intensified focus on the glycopeptide antibiotic vancomycin stems from its consideration as the "antibiotic of last resort" with respect to resistant bacterial strains ${ }^{42}$. Vancomycin functions by adhering to D-Ala-D-Ala sequences within the bacterial cell wall of Gram-positive bacteria and inhibits the transpeptidase activity required for the assembly of peptidoglycan (Fig. 3). Several synthetic studies have focused on the mechanism of vancomycin action, including the role of membrane anchoring ${ }^{43}$, electrostatic interaction $s^{44}$, multimerization ${ }^{45-48}$, and the pathway by which resistance develops (i.e., bacterial cell wall mutation of D-Ala-D-Ala to D-Ala-D-lactate $)^{49,50}$. A recent investigation suggests that mimics of the vancomycin glycan may exert antibacterial activity by inhibition of the transglycosylase, rather than the transpeptidase ${ }^{51}$.

Erythromycin is a macrolide antibiotic that is also employed in the treatment of infections caused by Gram-positive bacteria and serves as a clinical alternative for patients allergic to penicillins ${ }^{52}$. Characterization of the polyketide synthase responsible for erythromycin biosynthesis has resulted in the generation of novel macrolide structures. Through continuing investigation of the modular synthase, potent new antibiotics may be discovered for the fight against resistant bacteria ${ }^{53}$.

Lipopolysaccharide is the main outer-membrane component of Gram-negative bacteria. Antibacterial agents often cause disruption of bacterial membranes and the release of lipopolysaccharide, which can trigger an acute inflammatory response that leads to Gram- negative sepsis ${ }^{54}$. The reducing terminal glycophospholipid of lipopolysaccharide (Lipid A) contains all of the structural elements necessary to cause septic shock, and Lipid A antagonists are therefore considered therapeutic targets.

Various strategies toward this perplexing therapeutic dilemma (bacterial infection vs. endotoxic shock) have been undertaken to date. Lipid A from Rhodobacter sphaeroides is nontoxic, and synthesis of a structural mimic was one avenue explored ${ }^{55}$. This construct served as a lipopolysaccharide antagonist and protected mice from lipopolysaccharide-induced lethality. A second strategy involves disruption of lipopolysaccharide biosynthesis using the cytosine monophosphate (CMP)-3-deoxy-D-manno-octulosonate (KDO) synthetase inhibitor $\beta-\mathrm{KDO}^{56}$. Since $\beta-\mathrm{KDO}$ itself is membraneimpermeable, a "pro-drug" $\beta$-KDO-Ala-Ala conjugate capable of membrane permeation was synthesized. Treatment with this construct resulted in accumulation of a Lipid A precursor and bacterial cell death. Another protocol employed Lipid A substrate analogs as inhibitors of the deacetylase in the biosynthetic pathway ${ }^{57}$.

Helicobacter pylori is the main cause of gastritis, ulcers, and stomach cancer in humans. This bacterium recognizes both NeuAccontaining oligosaccharides ${ }^{58}$ and the $\mathrm{Le}^{\mathrm{b}}$ blood $^{59}$ group on the gastric epithelium. As such, soluble $\mathrm{Le}^{\mathrm{b}}$ and $\mathrm{3}^{\prime}$-sialyllactose may serve as therapeutic agents, by competitively inhibiting bacterial attachment to the gastric cell layer ${ }^{60}$.

Recently, it has been shown that the $\mathrm{AB}_{5}$ Shiga toxin pentamer from Shigella dysenteriae could be neutralized by a multivalent carbohydrate construct ${ }^{61}$. The potent five-armed "STARFISH" ligand was designed based on the X-ray co-crystal structure of the toxin bound to a monomeric ligand. Notably, in vitro inhibition of Shiga toxin by the polyvalent molecule was increased six orders of magnitude compared with the monovalent ligand.

The GPI biosynthetic pathway in trypanosomes has also been investigated as a point of therapeutic intervention in African sleeping sickness ${ }^{62}$. Using synthetic substrates as probes, Smith and colleagues $^{63}$ found that 2-O-methylation of D-myo-inositol was tolerated by trypanosomal $\alpha 1,4$-mannosyltransferase, but not by the human enzyme. This and other differences distinguish the biosynthetic pathways in parasites and mammals, providing potential leads for the design of antiparasitic agents.

The neomycin class of aminoglycoside antibiotics interacts with the Escherichia coli 16S rRNA A-site, thereby disrupting the fidelity of protein translation, and causing eventual bacterial cell death ${ }^{64,65}$. However, like glycopeptide antibiotics, prolonged treatment with aminoglycosides results in bacterial resistance. In this case, rather than mutate the RNA target, bacteria have evolved enzymes to modify the aminoglycosides. Following acetylation, phosphorylation, or adenylylation by these resistance-causing enzymes, the aminoglycosides have drastically reduced affinity for their RNA targets, and can no longer exert their antibacterial effects ${ }^{66}$.

To overcome resistance, as well as to combat such side effects of aminoglycoside administration as oto- and nephrotoxicity, synthetic antibiotic analogs have been developed ${ }^{67-70}$. Aminoglycoside derivatives have been analyzed with respect to A-site rRNA affinity, antibiotic activity, disruption of protein translation, and inhibition of aminoglycoside-modifying enzymes. These studies have resulted in the identification of an aminoglycoside dimer with nanomolar Asite affinity that was also a competitive inhibitor of resistance geneencoding aminoglycoside $6^{\prime}$-acetyltransferase $\left[\mathrm{AAC}\left(6^{\prime}\right)\right]$-aminoglycoside $2^{\prime \prime}$-phosphorylase $\left(\operatorname{APH}\left(2^{\prime \prime}\right) ; \text { Fig. } 4\right)^{68}$. In the same vein, Mobashery ${ }^{69}$ has accomplished the synthesis of an original kanamycin A derivative that intrinsically "resists" phosphorylation. Design of dual-function constructs of this nature may be the strategy required to combat resistant pathogens in the future. Aminoglycosides may also prove useful for the disruption of oncogenic mRNA translation ${ }^{71}$. 


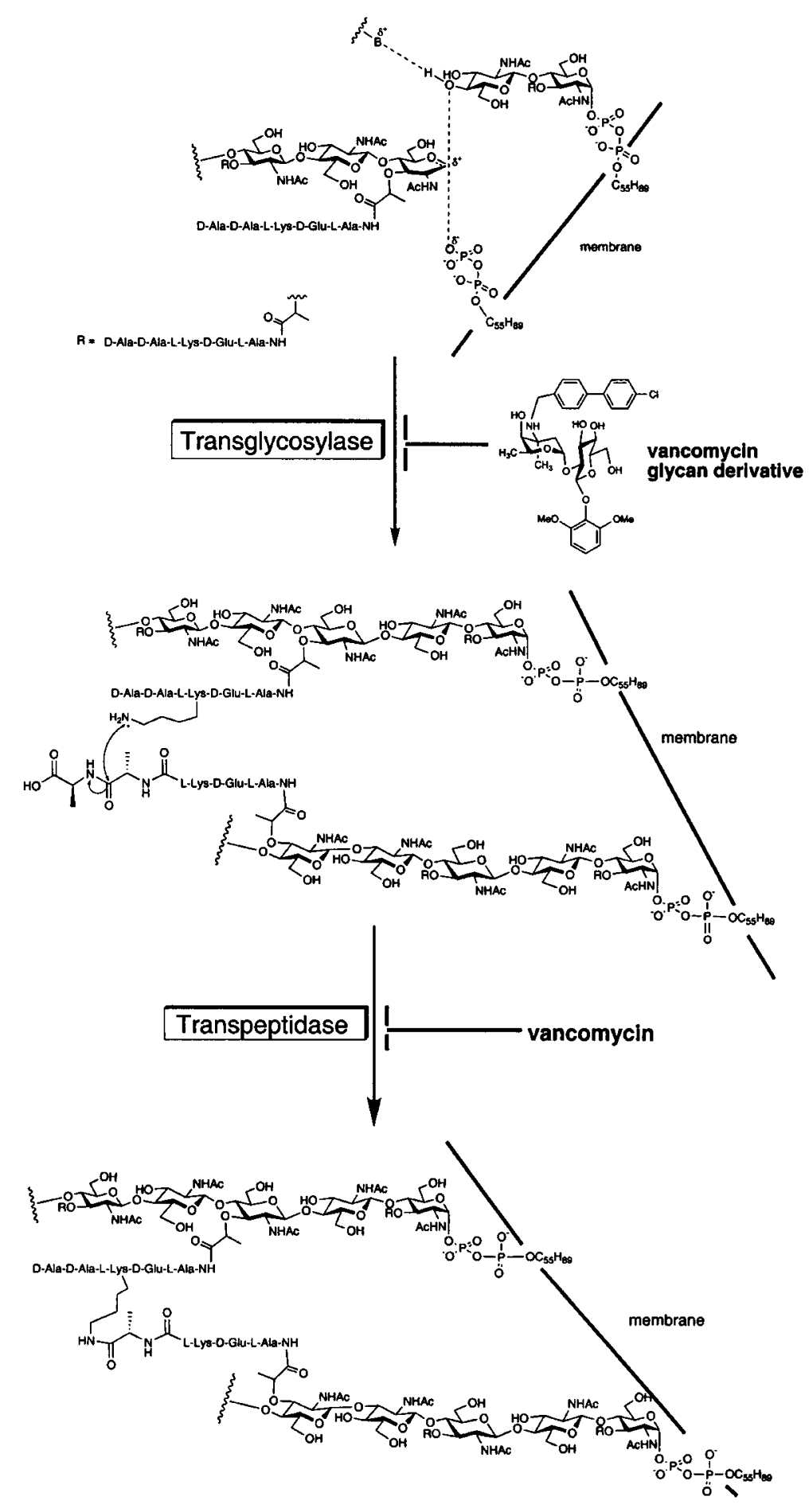

Figure 3. The biosynthesis of bacterial cell wall peptidoglycan involves both transglycosylase and transpeptidase activity. The antibiotic vancomycin inhibits the transpeptidase, and unique mimics of the vancomycin glycan inhibit the transglycosylase.

body with higher affinity than the natural $\alpha$-Gal epitope. In this strategy, polymeric $\alpha-G a l$ constructs effectively inhibit anti- $\alpha-$ Gal binding to pig kidney cells, and may have clinical potential ${ }^{72}$.

In contrast to evasion of the immune system, vaccines generated to glycoconjugates rely on the ability of the body to mount an immune response against a desired structure. The capsular polysaccharides present on invasive bacteria are prime targets for vaccine generation and have been used clinically in the treatment of bacterial meningitis and other diseases such as pneumonia and shigellosis ${ }^{73,74}$. For example, routine vaccination against Haemophilus influenzae type B with polysaccharide-protein conjugates is a highly effective treatment employed worldwide ${ }^{74}$. In a new report, a vaccine against Staphylococcus aureus has been developed to a polysaccharide antigen that is present on the bacteria during human infection, but not expressed during normal bacterial cell growth ${ }^{75}$. This strategy has proved effective in laboratory animals and is a candidate for clinical vaccination.

In general, because oligosaccharides themselves are T-cell-independent antigens and therefore serve as poor immunogens, several practical methods for conjugation to protein carriers (haptens) have been investigated. To date, most vaccines are generated to a panel of oligosaccharide structures present within a given capsular polysaccharide, a situation that raises the issue of whether the most relevant structure is adequately represented in the mixture of resulting polyclonal antibodies. In this respect, synthetic methods are valuable for the construction of a homogeneous oligosaccharide population before immunization.

In $\mathrm{CD}_{1}$-glycolipid antigen presentation to $\mathrm{T}$ cells, the immune response appears to be dependent on the presence of the glycan portion of the glycolipid, and this represents a possible alternative therapeutic avenue for vaccine development ${ }^{76,77}$. In this context, various polysialyltransferases from neuroinvasive bacteria can be employed to synthesize polysialylated glycolipids ${ }^{78,79}$. If coupled to $\mathrm{CD}_{1}$-mediated $\mathrm{T}$-cell activation, new vaccines may be developed to eradicate specific cancers or bacteria by the induction of killer T cells ${ }^{80}$.

Glycoconjugate vaccines are also emerging as a therapy in the battle against specific cancers ${ }^{7}$. Cell surface glycans are often altered or truncated in cancerous states as a result of a change in the regulation of specific glycosyltransferase genes. Interestingly, although glycosphingolipids are present on both normal tissue and tumors,

\section{The immune system as a therapeutic target}

As human organ donors are generally in short supply, animal tissues (especially those of porcine origin) have been suggested as an alternative solution; however, hyperacute rejection of foreign tissues from the human body currently precludes this practice. The discovery that the immune response against $\alpha$-galactosyl ( $\alpha$-Gal) epitopes present on xenotransplants is the primary cause of rejection has led to research into evasion of the immune system using synthetic constructs $^{72}$. Two general therapies have been undertaken. The first involves depleting human blood of anti- $\alpha$-Gal antibodies by passage through an $\alpha-G a l$ affinity matrix. The other is to construct soluble $\alpha$-Gal containing oligosaccharides that bind to the anti- $\alpha$-Gal anti- they act as immunogens only when present on the surface of tumor cells. Consequently, immunization to these antigenic determinants can provide vaccines useful in the eradication of cancerous cells from the body ${ }^{81}$. Notably, the fully synthetic Globo $\mathrm{H}$ vaccine has been shown to be immunogenic in humans for the treatment of prostate cancer (Fig. 5) ${ }^{82}$. Furthermore, the synthetic sialyl- $\mathrm{T}_{\mathrm{n}}$ vaccine for breast and ovarian cancers ${ }^{83}$ is highly target-specific, and its use correlates with prolonged survival in human clinical trials.

An alternative strategy involves vaccination with antibodies that, though generated by immunization to peptide antigens, cross-react with cell surface carbohydrate epitopes ${ }^{84}$. Numerous naturally occurring glycoconjugates, such as Reishi polysaccharides, also act as 

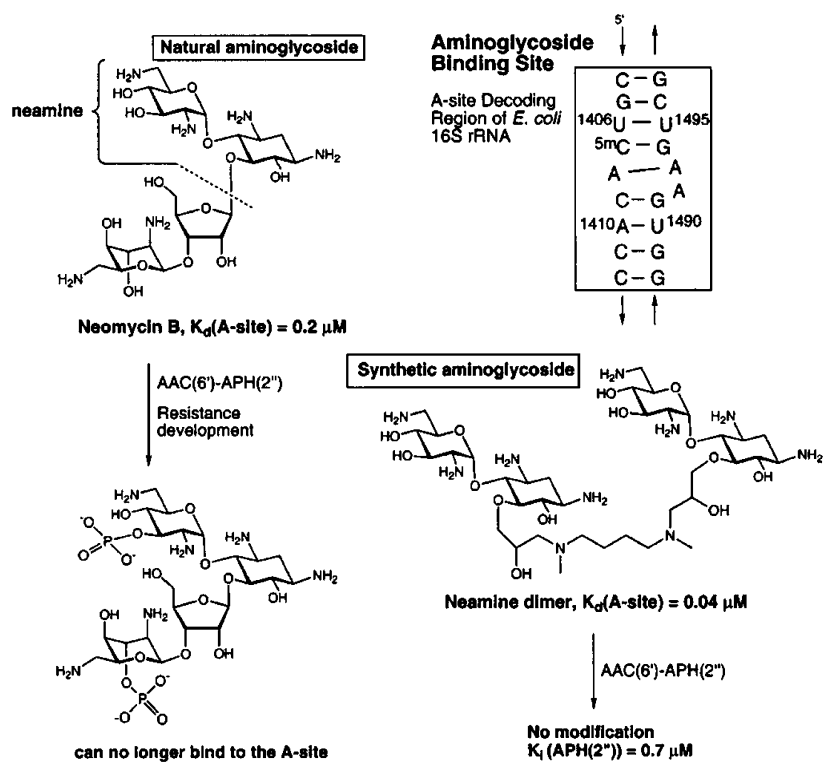

Figure 4. Aminoglycosides exert their antibiotic effects through binding of the bacterial 16S rRNA A-site. The neamine dimer pictured binds to the A-site with high affinity, and also inhibits the APH(2") activity of the resistance-causing bacterial enzyme $\operatorname{AAC}\left(6^{\prime}\right)-\operatorname{APH}\left(2^{\prime \prime}\right)$, thereby serving as a dual-function ligand in the fight against emerging resistant bacterial strains.

immunomodulators, although their mechanism of action is unknown ${ }^{85}$. Understanding the molecular mechanism of their function may lead to development of new carbohydrate-based therapeutics.

\section{Other targets}

Inhibitors of glycosyltransferases and glycosidases also have proven use as therapeutic agents. For example, treatment with $\mathrm{N}$-butyldeoxynojirimycin (NBJ) prevents the accumulation of ganglioside $\mathrm{G}_{\mathrm{M} 2}$ in the brain, by serving as a glucosyltransferase inhibitor (Fig. 6) ${ }^{86,87}$. As such, it has proven useful in the treatment of Tay-Sachs disease and may provide a general strategy for treating other sphingolipidoses ${ }^{88}$. The potent inhibition by NBJ of $\alpha$-glucosidases I and II probably accounts for its effectiveness in inhibiting HIV replication in vitro.

The inflammatory response employs the selectin family of carbohydrate-binding proteins in its early stages of leukocyte recruitment. Selectin-carbohydrate interactions have been extensively reviewed $^{89}$, and the development of inhibitors of selectin-ligand interactions generally relies on mimicking the sialyl-Lewis $\mathrm{x}$ $\left(\mathrm{sLe}^{\mathrm{x}}\right)$-selectin interaction in either mono- or polyvalent form. One class of cell adhesion inhibitors induces the proteolysis of L-selectin from the surface of leukocytes ${ }^{90}$. Alternative strategies toward antiinflammatory agents have taken the form of sLe ${ }^{\mathrm{x}}-\mathrm{RGD}$ (Arg-GlyAsp) conjugates that recognize both selectins and integrins ${ }^{91}$, or the inhibition of mannose phosphate receptors ${ }^{92}$. Furthermore, di-Le ${ }^{\mathrm{x}}$ has entered clinical trials in rheumatoid arthritis patients ${ }^{93}$. In the same vein, it has been shown that a sLe ${ }^{\mathrm{x}}$ glycosylated complement inhibitory protein (sCR1) inhibits both complement activation and selectin-mediated platelet-leukocyte interactions, thus reducing the level of neuronal injury due to reperfusion in stroke ${ }^{94}$.

Heparin is a heavily sulfated glycopolymer, employed for decades as an anticoagulant, that functions by inhibiting thrombin and Factor Xa through recognition of antithrombin III. However, administering large and heterogeneous heparin preparations can lead to detrimental side effects because of unfavorable interactions with blood vessel components. Recently, structurally defined heparin mimics with antithrombin activity similar to natural
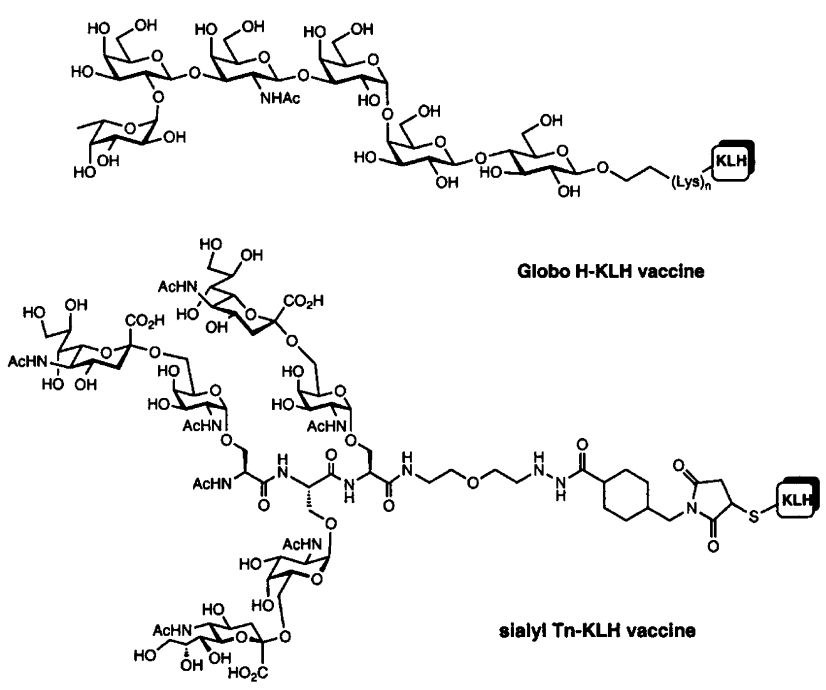

Figure 5. Keyhole limpet hemocyanin (KLH) conjugates of the Globo $H$ and sialyl- $T_{n}$ cancer antigens serve as effective vaccines against prostate and breast cancers, respectively.

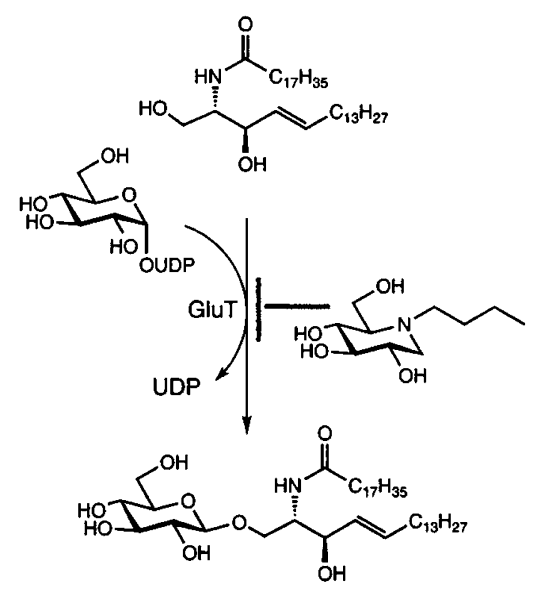

Figure 6. $\mathbf{N}$-butyldeoxynojirimicin inhibits the glucosyltransferase involved in sphingolipid biosynthesis, and serves as a therapeutic agent in the treatment of Tay-Sachs disease.

heparin, but with reduced side effects, have been obtained through chemical synthesis ${ }^{95,96}$.

Glycoproteins also have clinical utility, the prime example being erythropoietin $(\mathrm{EPO})^{97}$, which is widely used as a treatment for anemia associated with renal failure, chemotherapy, and 3 '-azidothymidine (AZT) administration. Interaction of EPO with its receptor (EpoR) stimulates erythropoeisis, resulting in the production of red blood cells. Studies of EPO have revealed that glycosylation, and especially the presence of sialic acid, is required for its normal function in vivo ${ }^{98,99}$. Currently, EPO must be administered intravenously or by subcutaneous injection, and agents that act similarly but are more conveniently delivered are under investigation. Toward this end, phage display techniques have identified short peptides that mimic the action of EPO by binding with high affinity to EpoR $\mathrm{R}^{100,101}$.

\section{Future directions}

In the past decade, glycoconjugate research has yielded an abundance of medicinally relevant information. New methods have eased the synthesis of complex oligosaccharides, and techniques for the facile synthesis of other glycoconjugates are rapidly developing. From a synthetic point of view, availability of an enzyme for each glycosidic 
linkage or an automated method to obtain any desired glycoconjugate would be advantageous. Furthermore, control of glycoprotein glycoform heterogeneity remains a problem and results in a poor understanding of the contribution of carbohydrates to protein structure and function. The development of methods for the construction of homogeneous glycoproteins is relevant not only from an academic standpoint, but also for large-scale production processes and marketing as pharmaceutical agents. Current synthetic efforts in this area have significantly advanced the field toward this end. Moreover, remodeling of cell surfaces employing glycosyltransferase strategies ${ }^{102}$ or by exploiting biosynthetic pathways ${ }^{103}$ may provide new insights into the function of cell surface glycoconjugates.

Therapeutically, it will be necessary to identify antibacterial agents that can evade resistant bacteria, because the clock on existing antibiotics is ticking. New strategies toward antibiotic agents are in high demand, especially those in which multiple mechanisms of action contribute to efficacy. In this area, the most significant results have been derived from the aminoglycoside antibiotics, which show promise as both RNA-binding molecules as well as therapeutics that avoid modification by resistance-causing enzymes. New antibiotics with novel function may also be discovered through manipulation of biosynthesis involving modular polyketide synthases.

Further evaluation of glycoconjugate vaccines will also be necessary, in light of the fact that great success has already been achieved in eradication of specific cancers. It is remarkable that the synthetic carbohydrate vaccines induce little undesirable response, even though the carbohydrate structures employed for immunization are present ubiquitously.

As our understanding of carbohydrate-mediated biological recognition processes advances to the molecular level, new carbohydrate-based therapies employing existing glycoconjugates and carbohydrate mimetics (noncarbohydrate organic molecules that resemble carbohydrates) will become readily available and widely utilized medicinal agents.

1. Varki, A. Biological roles of oligosaccharides: all of the theories are correct. Glycobiology 3, 97-130 (1993).

2. Dwek, R.A. Glycobiology: toward understanding the function of sugars. Chem. Rev. 96, 683-720 (1996).

3. Sears, P. \& Wong, C.-H. Enzyme action in glycoprotein synthesis. Cell. Mol. Life Sci. 54, 223-252 (1998).

4. Sears, P. \& Wong, C.-H. Carbohydrate mimetics: a new strategy for tackling the problem of carbohydrate-mediated biological recognition. Angew. Chem. Int. Ed. Engl. 38, 2300-2324 (1999).

5. Jacob, G.S. Glycosylation inhibitors in biology and medicine. Curr. Biol. 5, 605-611 (1995)

6. Kansas, G.S. Selectins and their ligands: current concepts and controversies. Blood 88, 3259-3287 (1996).

7. Hakomori, S.-i. \& Zhang, Y. Glycosphingolipid antigens and cancer therapy. Chem. Biol. 4, 97-104 (1997).

8. Nicolaou, K.C., Boddy, C.N.C., Brase, S. \& Winssinger, N. Chemistry, biology, and medicine of the glycopeptide antibiotics. Angew. Chem. Int. Ed. Engl. 38, 2096-2152 (1999).

9. Wessel, H.P. Heparinoid mimetics. In Topics in current chemistry. Glycoscience: synthesis of substrate analogs and mimetics (eds Driguez, H. \& Thiem, J.) 215-239 (Springer-Verlag, Berlin; 1997).

10. Englund, P.T. The structure and biosynthesis of glycosyl phosphatidylinositol protein anchors. Annu. Rev. Biochem. 62, 121-138 (1993)

11. Fukuda, M. Cell surface carbohydrates: cell-type specific expression. In Molecular glycobiology (eds Fukuda, M. \& Hindsgaul, O.) 1-52 (IRL Press, Oxford, UK; 1994)

12. Hart, G.W. Dynamic $O$-linked glycosylation of nuclear and cytoskeletal proteins. Annu. Rev. Biochem. 66, 315-335 (1997).

13. Fukuda, M.N., Sasaki, H., Lopez, L. \& Fukuda, M. Survival of recombinant erythropoeitin in the circulation: the role of carbohydrates. Blood 73, 84-89 (1989).

14. Jenkins, N., Parekh, R.B. \& James, D.C. Getting the glycosylation right: implications for the biotechnology industry. Nat. Biotechnol. 14, 975-981 (1996).

15. Schachter, $\mathrm{H}$. Biosynthetic controls that determine the branching and microheterogeneity of protein-bound oligosaccharides. Biochem. Cell Biol. 64 163-181 (1986).

16. Wong, C.-H., Halcomb, R.L., Ichikawa, Y. \& Kajimoto, T. Enzymes in organic synthesis: application to the problems of carbohydrate recognition (parts 1 and 2). Angew. Chem. Int. Ed. Engl. 34, 412-432; 521-546 (1995)

17. Gijsen, H.J.M., Qiao, L., Fitz, W. \& Wong, C.-H. Recent advances in the chemoenzymatic synthesis of carbohydrates and carbohydrate mimetics. Chem. Rev. 96, 443-473 (1996).
18. Koeller, K.M. \& Wong, C.-H. Synthesis of complex carbohydrates and glycoconjugates: enzyme-based and programmable one-pot strategies. Chem. Rev., in press (2000).

19. Koizumi, S., Endo, T., Tabata, K. \& Ozaki, A. Large-scale production of UDPgalactose and globotriose by coupling metabolically engineered bacteria. Nat. Biotechnol. 16, 847-850 (1998).

20. Witte, K., Sears, P., Martin, R. \& Wong, C.-H. Enzymatic glycoprotein synthesis: preparation of ribonuclease glycoforms via enzymatic glycopeptide condensation and glycosylation. J. Am. Chem. Soc. 119, 2114-2118 (1997).

21. Gewehr, M. \& Kunz, H. Comparative lipase-catalyzed hydrolysis of ethylene glycol derived esters. The 2-methoxyethyl ester as a protective group in peptide and glycopeptide synthesis. Synthesis 1499-1511 (1997).

22. Takayama, S., Livingston, P.O. \& Wong, C.-H. Synthesis of the melanoma-associated ganglioside 9-O-acetyl $\mathrm{GD}_{3}$ through regioselective enzymatic acetylation of $\mathrm{GD}_{3}$ using subtilisin. Tetrahedron Lett. 37, 9271-9274 (1996).

23. Machajewski, T.D. \& Wong, C.-H. The catalytic asymmetric aldol reaction Angew. Chem. Int. Ed. Engl. 39, 1352-1374 (2000).

24. Burkart, M.D., Izumi, M. \& Wong, C.-H. Enzymatic regeneration of 3 '-phosphoadenosine-5'-phosphosulfate using aryl sulfotransferase for the preparative enzymatic synthesis of sulfated carbohydrates. Angew. Chem. Int. Ed. Engl. 38, 2747-2750 (1999)

25. Shin, Y. et al. Fmoc-based synthesis of peptide ${ }^{\alpha}$ thioesters: application to the total chemical synthesis of a glycoprotein by native chemical ligation. J. Am. Chem. Soc. 121, 11684-11689 (1999).

26. Tolbert, T.J. \& Wong, C.-H. Intein-mediated synthesis of proteins containing carbohydrates and other molecular probes. J. Am. Chem. Soc. 122, 5421-5428 (2000).

27. Wang, L.-X. et al. Combined chemical and enzymatic synthesis of a C-glycopeptide and its inhibitory activity toward glycoamidases. J. Am. Chem. Soc. 119 11137-11146 (1997)

28. Zhang, Z. et al. Programmable one-pot oligosaccharide synthesis. J. Am. Chem. Soc. 121, 734-753 (1999).

29. Meanwell, N.A. \& Krystal, M. Taking aim at a moving target-inhibitors of influenza virus part 1: virus adsorption, entry, and uncoating. Drug Discovery Today 1 316-387 (1996).

30. Meanwell, N.A. \& Krystal, M. Taking aim at a moving target-inhibitors of influenza virus part 2: viral replication, packaging and release. Drug Discovery Today 1, 388-397 (1996)

31. Mammen, M., Choi, S.-K. \& Whitesides, G.M. Polyvalent interactions in biologica systems: implications for design and use of multivalent ligands and inhibitors. Angew. Chem. Int. Ed. Engl. 37, 2754-2794 (1998).

32. Kamitakahara, H. et al. A lysoganglioside/poly-L-glutamic acid conjugate as a picomolar inhibitor of influenza hemagglutinin. Angew. Chem. Int. Ed. Engl. 37 1524-1528 (1998).

33. von Itzstein, $M$. et al. Rational design of potent sialidase-based inhibitors of influenza virus replication. Nature $\mathbf{3 6 3}, 418-423$ (1993).

34. Kim, C. U. et al. Influenza neuraminidase inhibitors possessing a novel hydrophobic interaction in the enzyme active site: design, synthesis, and structural analysis of carbocyclic sialic acid analogues with potent anti-influenza activity. J. Am. Chem. Soc. 119, 681-690 (1997).

35. Harouse, J. M. et al. Inhibition of entry of HIV-1 in neural cell lines by antibodies against galactosyl ceramide. Science 253, 320-323 (1991).

36. Fantini, J. et al. Synthetic soluble analogs of galactosylceramide (GalCer) bind to the V3 domain of HIV-1 gp120 and inhibit HIV-1-induced fusion and entry. J. Biol. Chem. 272, 7245-7252 (1997).

37. Bertozzi, C.R., Cook, D.G., Kobertz, W.R., Gonzalez-Scarano, F. \& Bednarski, M.D. Carbon-linked galactosphingolipid analogs bind specifically to HIV-1 gp120. J. Am. Chem. Soc. 114, 10639-10641 (1992).

38. Zapp, M.L., Stern, S. \& Green, M.R. Small molecules that selectively block RNA binding of HIV-1 Rev protein inhibit Rev function and viral production. Cell $\mathbf{7 4}$ 969-978 (1993).

39. Park, W.K.C., Auer, M., Jaksche, H. \& Wong, C.-H. Rapid combinatorial synthesis of aminoglycoside antibiotic mimetics: use of a polyethylene glycol-linked amine and a neamine-derived aldehyde in multiple component condensation as a strategy for the discovery of new inhibitors of the HIV RNA Rev responsive element. J. Am. Chem. Soc. 118, 10150-10155 (1996).

40. Kirk, S.R., Luedtke, N.W. \& Tor, Y. Neomycin-acridine conjugate: a potent inhibitor of Rev-RRE binding. J. Am. Chem. Soc. 122, 980-981 (2000).

41. Karlsson, K.-A. Microbial recognition of target-cell glycoconjugates. Curr. Opin. Struc. Biol. 5, 622-635 (1995).

42. Williams, D.H. \& Bardsley, B. The vancomycin group of antibiotics and the fight against resistant bacteria. Angew. Chem. Int. Ed. Engl. 38, 1172-1193 (1999).

43. Sharman, G. J. et al. The roles of dimerization and membrane anchoring in activity of glycopeptide antibiotics against vancomycin-resistant bacteria. J. Am. Chem. Soc. 119, 12041-12047 (1997).

44. Xu, R., Greiveldinger, G., Marenus, L.E., Cooper, A. \& Ellman, J.A. Combinatoria library approach for the identification of synthetic receptors targeting vancomycin-resistant bacteria. J. Am. Chem. Soc. 121, 4898-4899 (1999).

45. Rao, J., Lahiri, J., Isaacs, L., Weis, R.M. \& Whitesides, G.M. A trivalent system from vancomycin:D-Ala-D-Ala with higher affinity than avidin-biotin. Science 280, 708-711 (1998)

46. Rao, J. \& Whitesides, G.M. Tight binding of a dimeric derivative of vancomycin with dimeric D-Ala-D-Ala. J. Am. Chem. Soc. 119, 10286-10290 (1997).

47. Sundram, U.N., Griffin, J.H. \& Nicas, T.I. Novel vancomycin dimers with activity against vancomycin-resistant enterococci. J. Am. Chem. Soc. 118 13107-13108 (1996)

48. Rao, J., Lahiri, J., Weis, R.M. \& Whitesides, G.M. Design, synthesis, and characterization of a high-affinity trivalent system derived from vancomycin and L-LysD-Ala-D-Ala. J. Am. Chem. Soc. 122:2698-2710 (2000).

49. Bugg, T.D.H. et al. Molecular basis for vancomycin resistance in Enterococcus faecium BM4147: biosynthesis of a depsipeptide peptidoglycan precursor by 
vancomycin resistance proteins VanH and VanA. Biochemistry 30, 10408-10415 (1990).

50. Walsh, C.T., Fisher, S.L., Park, I.-S., Prahalad, M. \& Wu, Z. Bacterial resistance to vancomycin: five genes and one missing hydrogen bond tell the story. Chem. Biol. 3, 21-28 (1996)

51. Ge, M. et al. Vancomycin derivatives that inhibit peptidoglycan biosynthesis without binding D-Ala-D-Ala. Science 284, 507-511 (1999).

52. Staunton, J. Combinatorial biosynthesis of erythromycin and complex polyketides. Curr. Opin. Chem. Biol. 2, 339-345 (1998).

53. Cane, D.E., Walsh, C.T. \& Khosla, C. Harnessing the biosynthetic code: combinations, permutations, and mutations. Science 282, 63-68 (1998).

54. Holst, O. Endotoxin antagonists: possible candidates for the treatment of gramnegative sepsis. Angew. Chem. Int. Ed. Eng. 34, 2000-2002 (1995).

55. Christ, W.J. et al. E5531, a pure endotoxin antagonist of high potency. Science 268, 80-83 (1995).

56. Hammond, S. M. et al. A new class of synthetic antibacterials acting on lipopolysaccharide biosynthesis. Nature 327, 730-732 (1987).

57. Jackman, J.E. et al. Antibacterial agents that target Lipid A biosynthesis in gramnegative bacteria. J. Biol. Chem. 275, 11002-11009 (2000)

58. Simon, P.M., Goode, P.L., Mobasseri, A. \& Zopf, D. Inhibition of Helicobacter pylori binding to gastrointestinal epithelial cells by sialic acid-containing oligosaccharides. Infect. Immun. 65, 750-757 (1997).

59. Boren, T., Falk, P., Roth, K.A., Larson, G. \& Normark, S. Attachment of Heliobacter pylori to human gastric epithelium mediated by blood group antigens. Science 262, 1892-1895 (1993).

60. Halcomb, R.L., Huang, H. \& Wong, C.-H. Solution- and solid-phase synthesis of inhibitors of $H$. pylori attachment and E-selectin-mediated leukocyte adhesion. $J$. Am. Chem. Soc. 116, 11315-11322 (1994).

61. Kitov, P.I. et al. Shiga-like toxins are neutralized by tailored multivalent carbohydrate ligands. Nature 403, 669-672 (2000)

62. Ferguson, M.A.J. et al. The GPI biosynthetic pathway as a therapeutic target for African sleeping sickness. Biochim. Biophys. Acta 1455, 327-340 (1999).

63. Smith, T.K. et al. Parasite and mammalian GPI biosynthetic pathways can be distinguished using synthetic substrate analogues. EMBO J. 16, 6667-6675 (1997).

64. Wong, C.-H., Hendrix, M., Priestly, E.S. \& Greenberg, W.A. Specificity of aminoglycoside antibiotics for the A-site of the decoding region of ribosomal RNA. Chem. Biol 5, 397-406 (1998).

65. Moazed, D. \& Noller, H.F. Interaction of antibiotics with functional sites in $16 \mathrm{~S}$ ribosomal RNA. Nature 327, 389-394 (1987).

66. Daigle, D.M., Hughes, D.W. \& Wright, G.D. Prodigious substrate specificity of $\mathrm{AAC}\left(6^{\prime}\right)-\mathrm{APH}\left(2^{\prime \prime}\right)$, an aminoglycoside antibiotic resistance determinant in enterococci and staphylococci. Chem. Biol. 6, 99-110 (1999).

67. Greenberg, W.A. et al. Design and synthesis of new aminoglycoside antibiotics containing neamine as an optimal core structure: correlation of antibiotic activity with in vitro inhibition of translation. J. Am. Chem. Soc. 121, 6527-6541 (1999).

68. Sucheck, S.J. et al. Design of bifunctional antibiotics that target bacterial rRNA and inhibit resistance-causing enzymes. J. Am. Chem. Soc. 122, 5230-5231 (2000).

69. Haddad, J., Vakulenko, S. \& Mobashery, S. An antibiotic cloaked by its own resistance mechanism. J. Am. Chem. Soc. 121, 11922-11923 (1999).

70. Ecker, D.J. \& Griffey, R.H. RNA as a small-molecule drug target: doubling the value of genomics. Drug Discovery Today 4, 420-429 (1999).

71. Sucheck, S.J., Greenberg, W.A., Tolbert, T.J. \& Wong, C.-H. Design of small molecules that recognize RNA: development of aminoglycosides as potential antitumor agents that target oncogenic RNA sequences. Angew. Chem. Int. Ed. Eng. 39, 1080-1084 (2000).

72. Wang, J.-Q. et al. Enhanced inhibition of human anti-gal antibody binding to mammalian cells by synthetic $\alpha$-gal epitope polymers. J. Am. Chem. Soc. 121, 8174-8181 (1999).

73. Sood, R.K., Fattom, A., Pavliak, V. \& Naso, R.B. Capsular polysaccharide-protein conjugate vaccines. Drug Discovery Today 1, 381-387 (1996)

74. Robbins, J.B., Schneerson, R., Szu, S.C. \& Pozsgay, V. Bacterial polysaccharide-protein conjugate vaccines. Pure Appl. Chem. 71, 745-754 (1999).

75. McKenney, D. et al. Broadly protective vaccine for Staphylococcus aureus based on an in vivo-expressed antigen. Science 284, 1523-1527 (1999).

76. Moody, D.B. et al. Structural requirements for glycolipid antigen recognition by CD1b-restricted T cells. Science 278, 283-286 (1997).
77. Burdin, N. \& Kronenberg, M. $C_{1}$-mediated immune responses to glycolipids. Curr. Opin. Immunol. 11, 326-331 (1999).

78. Cho, J.-W. \& Troy, F.A. Polysialic acid engineering: synthesis of polysialylated neoglycosphingolipids by using the polysialytransferase from neuroinvasive Escherichia coli K1. Proc. Natl. Acad. Sci. USA 91, 11427-11431 (1994).

79. Shen, G.-J., Datta, A.K., Izumi, M., Koeller, K.M. \& Wong, C.-H. Expression of $\alpha 2,8 / 2,9$-polysialyltransferase from Escherichia coli K92. J. Biol. Chem. 274, 35139-35146 (1999).

80. Kawano, $\mathrm{T}$. et al. CD1d-restricted and TCR-mediated activation of $\mathrm{V}_{\alpha} 14$ NKT cells by glycosylceramides. Science 278, 1626-1629 (1997).

81. Danishefsky, S.J. \& Allen, J.R. From the laboratory to the clinic: a retrospective on fully synthetic carbohydrate-based anticancer vaccines. Angew. Chem. Int. Ed. Engl. 39, 836-863 (2000).

82. Ragupathi, $\mathrm{G}$. et al. A fully synthetic Globo $\mathrm{H}$ carbohydrate vaccine induces a focused humoral response in prostate cancer patients: a proof of principle. Angew. Chem. Int. Ed. Engl. 38, 563-566 (1999)

83. Sandmaier, B.M. et al. Evidence of a cellular immune response against sialyl- $T_{n}$ in breast and ovarian cancer patients after high-dose chemotherapy, stem cell rescue, and immunization with theratope $\mathrm{ST}_{\mathrm{n}}-\mathrm{KLH}$ cancer vaccine. J. Immunother 22, 54-66 (1999).

84. Kieber-Emmons, T. et al. Vaccination with carbohydrate peptide mimotopes promotes anti-tumor responses. Nat. Biotechnol. 17, 660-665 (1999).

85. Wang, S.-Y. et al. The anti-tumor effect of Ganoderma lucidum is mediated by cytokines released from activated macrophages and T lymphocytes. Int. J. Cancer 70, 699-705 (1997).

86. Platt, F.M. et al. Prevention of lysosomal storage in Tay-Sachs mice treated with $\mathrm{N}$-butyldeoxynojirimycin. Science, 276, 428-431 (1997).

87. Kolter, T. A chemical concept for the treatment of Tay-Sachs disease. Angew. Chem. Int. Ed. Engl. 36, 1955-1959 (1997).

88. Kolter, T. \& Sandhoff, K. Sphingolipids - their metabolic pathways and the pathobiochemistry of neurodegenerative diseases. Angew. Chem. Int. Ed. Engl. 38, 1532-1568 (1999).

89. Simanek, E.E., McGarvey, G.J., Jablonowski, J.A. \& Wong, C.-H. Selectin-carbohydrate interactions: from natural ligands to designed mimetics. Chem. Rev. $\mathbf{9 8}$ 833-862 (1998).

90. Gordon, E.J., Sanders, W.J. \& Kiessling, L.L. Synthetic ligands point to cell surface strategies. Nature 392, 30-31 (1998).

91. Sprengard, U., Kretzschmar, G., Bartnik, E., Huls, C. \& Kunz, H. Synthesis of an RGD-sialyl-Lewis $\times$ glycoconjugate: a new highly active ligand for P-selectin. Angew. Chem. Int. Ed. Engl. 34, 990-993 (1995).

92. St. Hilaire, P.M. \& Meldal, M. Glycopeptide and oligosaccharide libraries. Angew. Chem. Int. Ed. Engl. 39, 1162-1179 (2000).

93. Ochi, T. et al. Therapeutic effect of intradermal injections with difucosyl lactosamine (dimeric $\mathrm{Le}^{\mathrm{x}}$ ) on patients with rheumatoid arthritis. J. Rheumatol. 20, 2038-2045 (1993).

94. Huang, J. et al. Neuronal protection in stroke by an sLe $\mathrm{s}^{\mathrm{x}}$-glycosylated complement inhibitory protein. Science 285, 595-599 (1999).

95. Petitou, M. et al. First synthetic carbohydrates with the full anticoagulant properties of heparin. Angew. Chem. Int. Ed. Engl. 37, 3009-3014 (1998).

96. Petitou, M. et al. Synthesis of thrombin-inhibiting heparin mimetics without side effects. Nature 398, 417-422 (1999).

97. Cazzola, M., Mercuriali, F. \& Brugnara, C. Use of recombinant human erythropoietin outside the setting of uremia. Blood 89, 4248-4267 (1997).

98. Liu, D.T.-Y. Glycoprotein pharmaceuticals: scientific and regulatory considerations, and the US orphan drug act. Trends Biotechnol. 10, 114-120 (1992).

99. Geisow, M.J. Glycoprotein glycans-roles and controls. Trends Biotechnol. 10, 333-335 (1992)

100. Wrighton, N.C. et al. Small peptides as potent mimetics of the protein hormone erythropoietin. Science 273, 458-463 (1996).

101. Livnah, O. et al. Functional mimicry of a protein hormone by a peptide agonist: the EPO receptor complex at $2.8 \AA \AA$. Science 273, 464-471 (1996).

102. Srivastava, G., Kaur, K.J., Hindsgaul, O. \& Palcic, M.M. Enzymatic transfer of a preassembled trisaccharide antigen to cell surfaces using a fucosyltransferase. J. Biol. Chem. 267, 22356-22361 (1992).

103. Mahal, L.K., Yarema, K.J. \& Bertozzi, C.R. Engineering chemical reactivity on cell surfaces through oligosaccharide biosynthesis. Science 276, 1125-1128 (1997). 